TRANSACTIONS OF THE

AMERICAN MATHEMATICAL SOCIETY

Volume 356, Number 12, Pages 4897-4908

S 0002-9947(04)03534-2

Article electronically published on January 16, 2004

\title{
A LOCAL LIMIT THEOREM FOR CLOSED GEODESICS AND HOMOLOGY
}

\author{
RICHARD SHARP
}

\begin{abstract}
In this paper, we study the distribution of closed geodesics on a compact negatively curved manifold. We concentrate on geodesics lying in a prescribed homology class and, under certain conditions, obtain a local limit theorem to describe the asymptotic behaviour of the associated counting function as the homology class varies.
\end{abstract}

\section{INTRODUCTION}

Let $M$ be a compact smooth Riemannian manifold with first Betti number $k>0$ and with negative sectional curvatures. Suppose also that either $\operatorname{dim} M=2$ or that $M$ is $1 / 4$-pinched, i.e., the sectional curvatures all lie in an interval $[-\kappa,-\kappa / 4]$, for some $\kappa>0$. Such a manifold contains a countable infinity of prime closed geodesics. (We say that a closed geodesic is prime if it is not a nontrivial multiple of another closed geodesic.) In this paper we are interested in how these closed geodesics are distributed with respect to homology.

The homology group $H_{1}(M, \mathbb{Z})$ is isomorphic to $\mathbb{Z}^{k} \oplus$ Tor, where Tor is the (finite) torsion subgroup. In this paper, it will be convenient to consider the torsion-free part of the homology, $H_{1}(M, \mathbb{Z}) /$ Tor. We shall, in fact, assume that an isomorphism has been fixed and write $\mathbb{Z}^{k}$ instead of $H_{1}(M, \mathbb{Z}) /$ Tor.

For a typical (prime) closed geodesic $\gamma$ on $M$, let $l(\gamma)$ denote its length and $[\gamma] \in H_{1}(M, \mathbb{Z}) /$ Tor $=\mathbb{Z}^{k}$ the torsion-free part of its homology class. For $\alpha \in \mathbb{Z}^{k}$, define a counting function

$$
\pi(T, \alpha)=\#\{\gamma: l(\gamma) \leq T,[\gamma]=\alpha\} .
$$

Recently, several papers have studied the asymptotics of this function as $T \rightarrow \infty$. In particular, Anantharaman [1] and Pollicott and Sharp [17] have shown that there exist constants $C_{0}>0$, independent of $\alpha$, and $C_{n}(\alpha), n \geq 1$, such that, for any $N \geq 1$, we have the asymptotic expansion

$$
\pi(T, \alpha)=\frac{e^{h T}}{T^{k / 2+1}}\left(C_{0}+\frac{C_{1}(\alpha)}{T}+\frac{C_{2}(\alpha)}{T^{2}}+\cdots+\frac{C_{N}(\alpha)}{T^{N}}+O\left(\frac{1}{T^{N+1}}\right)\right),
$$

where $h>0$ denotes the topological entropy of the geodesic flow over $M$. (In fact, the expansion in [17] contains some extra terms corresponding to powers of $T^{-1 / 2}$; a more careful analysis, as carried out in [11], shows that these terms vanish.) Furthermore, Kotani [11, has studied the dependence of the coefficients $C_{n}(\alpha)$ on

Received by the editors March 28, 2003 and, in revised form, June 6, 2003.

2000 Mathematics Subject Classification. Primary 37C27, 37C30, 37D20, 37D40, 53C22.

The author was supported by an EPSRC Advanced Research Fellowship. 
$\alpha=\left(\alpha_{1}, \ldots, \alpha_{k}\right)$, showing that they may be expressed as polynomials of degree $2 n$ in $\alpha_{1}, \ldots, \alpha_{k}$. In the special case of manifolds of constant negative curvature, the expansion (0.1) was obtained by Phillips and Sarnak [14] and, independently, Katsuda and Sunada 9] obtained the leading term. For manifolds of variable negative curvature (without the pinching condition) the leading term of $(0.1)$ was obtained by Katsuda [8, Lalley [12] and Pollicott [15]. Analogous results for manifolds with cusps have been obtained by Epstein $[6$ and Babillot and Peigné 3$]$.

In this note, we take a slightly different view and address the question of the behaviour of $\pi(T, \alpha)$ when $\alpha$ is allowed to vary independently of $T$. We obtain the following "local limit theorem".

Theorem 1. Let $M$ be a compact smooth Riemannian manifold with first Betti number $k>0$ and with negative sectional curvatures. Suppose also that either $\operatorname{dim} M=2$ or that $M$ is 1/4-pinched. Then there exists a symmetric positive definite real matrix $\mathcal{D}$ such that

$$
\lim _{T \rightarrow \infty}\left|\frac{h \sigma^{k} T^{k / 2+1}}{e^{h T}} \pi(T, \alpha)-\frac{1}{(2 \pi)^{k / 2}} e^{-\left\langle\alpha, \mathcal{D}^{-1} \alpha\right\rangle / 2 T}\right|=0,
$$

uniformly in $\alpha \in \mathbb{Z}^{k}$, where $\sigma>0$ satisfies $\sigma^{2 k}=\operatorname{det} \mathcal{D}$.

Here, $\langle\cdot, \cdot\rangle$ denotes the usual inner product $\langle x, y\rangle=x_{1} y_{1}+\cdots+x_{k} y_{k}$. As a particular consequence, we recover the leading term of the expansion (0.1), with $C_{0}=h^{-1} \sigma^{-k}(2 \pi)^{-k / 2}$. Theorem 1 appears not to have been stated even for manifolds of constant negative curvature; although, in that case, the result can be easily deduced from the analysis contained in [14.

Remarks. (i) If we take the torsion part of $H_{1}(M, \mathbb{Z})$ into account, then we need to modify Theorem 1 to read

$$
\lim _{T \rightarrow \infty}\left|\frac{h \sigma^{k} T^{k / 2+1}}{e^{h T}} \pi(T, \alpha)-\frac{1}{(\# \text { Tor })(2 \pi)^{k / 2}} e^{-\left\langle\alpha_{F}, \mathcal{D}^{-1} \alpha_{F}\right\rangle / 2 T}\right|=0,
$$

uniformly in $\alpha \in H_{1}(M, \mathbb{Z})$, where $\alpha_{F} \in \mathbb{Z}^{k}$ denotes the torsion-free part of $\alpha \in$ $H_{1}(M, \mathbb{Z})$.

(ii) In Kotani's formula for the term $C_{n}(\alpha) / T^{n}$ in (0.1), the highest power of $\alpha$ makes a contribution

$$
\frac{1}{(2 \pi)^{k / 2} h \sigma^{k}} \frac{1}{n !}\left(-\frac{\left\langle\alpha, \mathcal{D}^{-1} \alpha\right\rangle}{2 T}\right)^{n} .
$$

As observed by Kotani in [11, formally summing these contributions gives the expression $e^{-\left\langle\alpha, \mathcal{D}^{-1} \alpha\right\rangle / 2 T} /(2 \pi)^{k / 2} h \sigma^{k}$.

Theorem 1 should be compared with the results on homology classes varying linearly in $T$ obtained by Lalley [12] and Babillot and Ledrappier [2]. Using these results, one can show that, for $\delta>0$ sufficiently small,

$$
\lim _{T \rightarrow \infty} \sup _{\|\alpha\| \leq \delta T}\left|\frac{T^{k / 2+1}}{C(\alpha / T) e^{H(\alpha / T) T}} \pi(T, \alpha)-1\right|=0
$$

where $H(x)$ is an entropy function satisfying $H(0)=h, \nabla H(0)=0$ and $\nabla^{2} H(0)=$ $-\mathcal{D}^{-1}$, and where $C(x)$ is continuous with $C(0)=C_{0}$. On the other hand, Theorem 
1 is equivalent to

$$
\lim _{T \rightarrow \infty} \sup _{\|\alpha\| \leq \delta T}\left|\frac{h \sigma^{k} T^{k / 2+1}}{e^{h T}} \pi(T, \alpha)-\frac{1}{(2 \pi)^{k / 2}} e^{-\left\langle\alpha, \mathcal{D}^{-1} \alpha\right\rangle / 2 T}\right|=0
$$

(as the supremum over $\|\alpha\|>\delta T$ clearly tends to zero). However, even though $H(x)=H(0)-\left\langle x, \mathcal{D}^{-1} x\right\rangle / 2+O\left(\|x\|^{3}\right)$, which gives

$$
\exp \{H(\alpha / T) T\}=\exp \left\{H(0) T-\left\langle\alpha, \mathcal{D}^{-1} \alpha\right\rangle / 2 T+O\left(\|\alpha\|^{3} / T^{2}\right)\right\},
$$

the presence of the third order terms means that one cannot deduce (0.3) from $(0.2)$.

The results of [2] and [12] do imply a central limit theorem: for $A \subset \mathbb{R}^{k}$,

$$
\lim _{T \rightarrow \infty} \frac{\#\{\gamma: l(\gamma) \leq T,[\gamma] / \sqrt{T} \in A\}}{\#\{\gamma: l(\gamma) \leq T\}}=\frac{1}{(2 \pi)^{k / 2} \sigma^{k}} \int_{A} e^{-\left\langle x, \mathcal{D}^{-1} x\right\rangle / 2} d x
$$

A key ingredient in the proof of Theorem 1 is an understanding of the analytic domain of a family of functions of a complex variable, called $L$-functions, indexed by the characters of $\mathbb{Z}^{k}$. In the next section, we shall define these functions and discuss their properties. In Section 2, we shall introduce a family of functions $\mathcal{S}_{T}(t), t \in[-\pi, \pi]^{k}$, obtained by summing a suitably weighted character $e^{i\langle t, \cdot\rangle}$ over all (multiple) closed geodesics of length at most $T$, and show that they are related to contour integrals of the corresponding $L$-functions. The results in Section 1 are then used to estimate the sums $\mathcal{S}_{T}(t)$. In Section 3, we shall use an approach adapted from [18] to transfer information from the $\mathcal{S}_{T}(t)$ to an auxiliary function $\psi(T, \alpha)$, which is essentially a weighted version of $\pi(T, \alpha)$, and obtain an analogue of Theorem 1 valid for $\psi(T, \alpha)$. In Section 4, we shall complete the proof of Theorem 1 by elementary arguments. In the final section, we shall discuss the application of our method to homologically full Anosov flows, giving a new proof of the first order asymptotic formula for $\pi(T, \alpha)$ (but without uniformity) in that case.

Notation. For given functions $A(T)$ and $B(T)$, we shall write $A(T) \sim B(T)$, as $T \rightarrow \infty$, if $\lim _{T \rightarrow \infty} A(T) / B(T)=1$, and $A(T)=O(B(T))$ if $|A(T)| \leq C B(T)$, for some constant $C>0$.

\section{1. $L$-FUNCTIONS}

In order to obtain our main result, we shall need to understand the analytic behaviour of a certain family of functions of a complex variable. We will identify the character group of $\mathbb{Z}^{k}$ with $[-\pi, \pi]^{k}$. For $t \in[-\pi, \pi]^{k}$, define

$$
L(s, t)=\prod_{\gamma}\left(1-e^{-s l(\gamma)+i\langle t,[\gamma]\rangle}\right)^{-1}
$$

where the product is taken over all prime closed geodesics $\gamma$. This converges for $R e(s)>h$ and has a meromorphic extension to a strictly larger half-plane [13.

It will be convenient to consider multiple closed geodesics $\gamma^{\prime}=\gamma^{n}, n \geq 1$. In this case we shall write $l\left(\gamma^{\prime}\right)=n l(\gamma),\left[\gamma^{\prime}\right]=n[\gamma]$, and $\Lambda\left(\gamma^{\prime}\right)=l(\gamma)$. (Note that $\Lambda$ is analogous to the von Mangoldt function in number theory.)

We are interested in the logarithmic derivative $L^{\prime}(s, t) / L(s, t)$ of $L(s, t)$. Whenever the summation converges, we have the identity

$$
\frac{L^{\prime}(s, t)}{L(s, t)}=-\sum_{\gamma^{\prime}} \Lambda\left(\gamma^{\prime}\right) e^{-s l\left(\gamma^{\prime}\right)+i\left\langle t,\left[\gamma^{\prime}\right]\right\rangle} .
$$


We shall make use of the properties of $L^{\prime}(s, t) / L(s, t)$ described by the following two propositions. These results were obtained in [17] and rely heavily on the techniques of Dolgopyat [4]. We write $U(\delta)=\{t:\|t\|<\delta\}$.

Proposition 1 ([17]). For all sufficiently small $\delta>0$ the following statements are true.

(i) There exists $\epsilon>0$ and an analytic function $s: U(\delta) \rightarrow(-\infty, h]$, satisfying $s(0)=h$ and $s(t)<h$ for $t \neq 0$, such that

$$
\frac{L^{\prime}(s, t)}{L(s, t)}+\frac{1}{s-s(t)}
$$

is analytic in $\operatorname{Re}(s)>h-\epsilon$.

(ii) There exists $\epsilon>0$ such that, for $t \notin U(\delta), L^{\prime}(s, t) / L(s, t)$ is analytic in $\operatorname{Re}(s)>h-\epsilon$.

Proposition $2([17])$. There exists $\epsilon>0, C>0$, and $0<\beta<1$, such that, for all $t \in[-\pi, \pi]^{k}$,

$$
\left|\frac{L^{\prime}(s, t)}{L(s, t)}\right| \leq C|\operatorname{Im}(s)|^{\beta},
$$

for $\operatorname{Re}(s)>1-\epsilon$ and $|\operatorname{Im}(s)| \geq 1$.

The function $s(t)$ enjoys the following properties.

Lemma 1. $\nabla s(0)=0$ and $\nabla^{2} s(0)$ is strictly negative definite.

We shall write $\mathcal{D}=-\nabla^{2} s(0)$ and define $\sigma>0$ by $\sigma^{2 k}=\operatorname{det} \mathcal{D}$. The next result is crucial for our subsequent analysis.

Proposition 3. There exists $\delta>0$ such that, for $t \in U(\delta \sigma \sqrt{T})$,

$$
\lim _{T \rightarrow \infty} e^{(s(t / \sigma \sqrt{T})-h) T}=e^{-\langle t, \mathcal{D} t\rangle / 2 \sigma^{2}} .
$$

Furthermore, $\left|e^{(s(t / \sigma \sqrt{T})-h) T}\right| \leq e^{-\langle t, \mathcal{D} t\rangle / 4 \sigma^{2}}$ and

$$
\left|e^{(s(t / \sigma \sqrt{T})-h) T}-e^{-\langle t, \mathcal{D} t\rangle / 2 \sigma^{2}}\right| \leq 2 e^{-\langle t, \mathcal{D} t\rangle / 4 \sigma^{2}} .
$$

Proof. Let $f(t)=e^{s(t)-h}$. Then $f(0)=1, \nabla f(0)=\nabla s(0)=0$, and $\nabla^{2} f(0)=$ $\nabla^{2} s(0)=-\mathcal{D}$. Applying Taylor's Theorem, we have that, for $\|t / \sigma \sqrt{T}\| \leq \delta$,

$$
f\left(\frac{t}{\sigma \sqrt{T}}\right)=1-\frac{\langle t, \mathcal{D} t\rangle}{2 \sigma^{2} T}+O\left(\frac{\|t\|^{3}}{T^{3 / 2}}\right)
$$

(where the implied constant is independent of $t$ ). The first statement follows from the identity $\lim _{T \rightarrow \infty}(1-x / T)^{T}=e^{-x}$.

Provided $\delta>0$ is sufficiently small, for $\|u\| \leq \delta$, we have

$$
\langle u, \mathcal{D} u\rangle / 2+O\left(\|u\|^{3}\right) \geq\langle u, \mathcal{D} u\rangle / 4 .
$$

Since $(1-x / T)^{T}<e^{-x}$, this gives us $|f(t / \sigma \sqrt{T})| \leq e^{-\langle t, \mathcal{D} t\rangle / 4 \sigma^{2}}$. Applying the triangle inequality, we obtain

$$
\begin{aligned}
\left|f(t / \sigma \sqrt{T})-e^{-\langle t, \mathcal{D} t\rangle / 2 \sigma^{2}}\right| & \leq e^{-\langle t, \mathcal{D} t\rangle / 4 \sigma^{2}}+e^{-\langle t, \mathcal{D} t\rangle / 2 \sigma^{2}} \\
& \leq 2 e^{-\langle t, \mathcal{D} t\rangle / 4 \sigma^{2}}
\end{aligned}
$$


Remark. The function $s(t)$ has an interpretation in terms of the thermodynamic formalism of the geodesic flow on $S M$. For a continuous function $G: S M \rightarrow \mathbb{R}$, define its pressure $P(G)=\sup _{\mu}\left\{h_{\mu}(\phi)+\int G d \mu\right\}$, where the supremum is taken over all probability measures invariant under the geodesic flow. We can define a (smooth) function $F: S M \rightarrow \mathbb{R}^{k}$ with the property that, for each closed geodesic

$\gamma, \int_{0}^{l(\gamma)} F(\gamma(t), \dot{\gamma}(t)) d t=[\gamma]$. Then $\mathbb{R}^{k} \ni z \mapsto P(\langle z, F\rangle)$ is real analytic and has an analytic extension to a neighbourhood of $\mathbb{R}^{k}$ in $\mathbb{C}^{k}$. We have that $s(t)=P(\langle i t, F\rangle)$ and that $\mathcal{D}=\left.\nabla^{2} P(\langle z, F\rangle)\right|_{z=0}[10]$, [19].

\section{Contour integration}

We shall now use the results on $L$-functions obtained in the preceding section to examine the behaviour of the summatory function

$$
\mathcal{S}_{T}(t)=\sum_{l\left(\gamma^{\prime}\right) \leq T}^{\prime} \Lambda\left(\gamma^{\prime}\right) e^{i\left\langle t,\left[\gamma^{\prime}\right]\right\rangle},
$$

as $T \rightarrow \infty$. (Here, the ' on the summation sign denotes that the terms with $l\left(\gamma^{\prime}\right)=T$ are counted with weight $1 / 2$.)

We begin by relating $\mathcal{S}_{T}(t)$ to $L^{\prime}(s, t) / L(s, t)$. This is achieved through the following lemma.

Lemma 2 ([20, p. 132], Effective Perron Formula). Define a function $\theta(y)$ by

$$
\theta(y)=\left\{\begin{array}{l}
0 \text { if } 0<y<1 \\
\frac{1}{2} \text { if } y=1 \\
1 \text { if } y>1
\end{array}\right.
$$

Then, uniformly for $d>0, R>0$,

$$
\left|\theta(y)-\frac{1}{2 \pi i} \int_{d-i R}^{d+i R} \frac{y^{s}}{s} d s\right|=O\left(\frac{y^{d}}{1+R|\log y|}\right) .
$$

Set $d=h+T^{-1}$ and $R=T^{K}$ (where $K>0$ will be chosen later). Applying Lemma 2 term-by-term to $-L^{\prime}(s, t) / L(s, t)$, we obtain

$$
\mathcal{S}_{T}(t)=\frac{1}{2 \pi i} \int_{d-i R}^{d+i R}\left(-\frac{L^{\prime}(s, t)}{L(s, t)}\right) \frac{e^{s T}}{s} d s+O\left(\sum_{\gamma^{\prime}} \frac{\Lambda\left(\gamma^{\prime}\right) e^{d T} e^{-d l\left(\gamma^{\prime}\right)}}{1+R\left|T-l\left(\gamma^{\prime}\right)\right|}\right) .
$$

We will estimate the big- $O$ term in this expression. First set $\epsilon=T^{-M}$ (where $M>0$ will be chosen later) and consider the terms for which $\left|T-l\left(\gamma^{\prime}\right)\right| \leq \epsilon$. We will use the following result contained in [16].

Proposition 4 ([16]). There exists $c<h$ such that

$$
\#\left\{\gamma^{\prime}: l\left(\gamma^{\prime}\right) \leq T\right\}=\int_{2}^{e^{h T}} \frac{1}{\log u} d u+O\left(e^{c T}\right) .
$$

As a consequence, we may write

$$
\#\left\{\gamma^{\prime}:\left|T-l\left(\gamma^{\prime}\right)\right| \leq \epsilon\right\}=\int_{e^{h T-h \epsilon}}^{e^{h T+h \epsilon}} \frac{1}{\log u} d u+O\left(e^{c T}\right)=O\left(\frac{\epsilon e^{h T}}{T}\right) .
$$


Furthermore, if $\left|T-l\left(\gamma^{\prime}\right)\right| \leq \epsilon$, then $e^{d T} e^{-d l\left(\gamma^{\prime}\right)}=O(1)$. Thus,

$$
\sum_{\left|T-l\left(\gamma^{\prime}\right)\right| \leq \epsilon} \frac{\Lambda\left(\gamma^{\prime}\right) e^{d T} e^{-d l\left(\gamma^{\prime}\right)}}{1+R\left|T-l\left(\gamma^{\prime}\right)\right|}=O\left(\frac{e^{h T}}{T^{M}}\right) .
$$

On the other hand,

$$
\sum_{\left|T-l\left(\gamma^{\prime}\right)\right|>\epsilon} \frac{\Lambda\left(\gamma^{\prime}\right) e^{d T} e^{-d l\left(\gamma^{\prime}\right)}}{1+R\left|T-l\left(\gamma^{\prime}\right)\right|} \leq \frac{e^{d T}}{R \epsilon} \sum_{\gamma^{\prime}} \Lambda\left(\gamma^{\prime}\right) e^{-d l\left(\gamma^{\prime}\right)}=O\left(\frac{e^{h T}}{T^{K-M-1}}\right),
$$

where we have used the estimate

$$
\left|\frac{L^{\prime}\left(h+T^{-1}, 0\right)}{L\left(h+T^{-1}, 0\right)}\right|=O(T) .
$$

Combining the estimates above, equation (2.1) becomes

$$
\mathcal{S}_{T}(t)=\frac{1}{2 \pi i} \int_{d-i R}^{d+i R}\left(-\frac{L^{\prime}(s, t)}{L(s, t)}\right) \frac{e^{s T}}{s} d s+O\left(\frac{e^{h T}}{T^{\min \{M, K-M-1\}}}\right) .
$$

Lemma 3. For all $N \geq 1$ we have the following estimates. (The implied constants are independent of $t$.)

(i) For $t \in U(\delta)$,

$$
\mathcal{S}_{T}(t)=\frac{e^{s(t) T}}{s(t)}+O\left(\frac{e^{h T}}{T^{N}}\right)
$$

(ii) For $t \notin U(\delta)$,

$$
\mathcal{S}_{T}(t)=O\left(\frac{e^{h T}}{T^{N}}\right) .
$$

Proof. Choose $h-\epsilon<c<h$ and let $\Gamma$ denote the contour formed by the rectangle with vertices at $d-i R, d+i R, c+i R$, and $c-i R$, oriented counterclockwise.

(i) Suppose that $t \in U(\delta)$. By Proposition 1(i) we can choose $c<s(t)$ so that, using the Residue Theorem,

$$
\frac{1}{2 \pi i} \int_{\Gamma}\left(-\frac{L^{\prime}(s, t)}{L(s, t)}\right) \frac{e^{s T}}{s} d s=\frac{e^{s(t) T}}{s(t)} .
$$

Using Proposition 2, we also have the following bounds:

(a)

$$
\left|\left(\int_{c+i R}^{d+i R}+\int_{c-i R}^{d-i R}\right)\left(-\frac{L^{\prime}(s, t)}{L(s, t)}\right) \frac{e^{s T}}{s} d s\right|=O\left(R^{\beta-1} e^{h T}\right)=O\left(\frac{e^{h T}}{T^{K(1-\beta)}}\right) ;
$$

(b)

$$
\left|\int_{c-i R}^{c+i R}\left(-\frac{L^{\prime}(s, t)}{L(s, t)}\right) \frac{e^{s T}}{s} d s\right|=O\left(R^{\beta} e^{c T}\right)=O\left(T^{\beta K} e^{c T}\right) .
$$

Combining this with (2.2) gives

$$
\mathcal{S}_{T}(t)=\frac{e^{s(t) T}}{s(t)}+O\left(\frac{e^{h T}}{T^{N}}\right)
$$

where

$$
N=\min \{M, K-M-1, K(1-\beta)\} .
$$

Since $K$ and $M$ are arbitrary, we may take $N$ as large as we please. 
(ii) Suppose that $t \notin U(\delta)$. Then, by Proposition 1(ii),

$$
\frac{1}{2 \pi i} \int_{\Gamma}\left(-\frac{L^{\prime}(s, t)}{L(s, t)}\right) \frac{e^{s T}}{s} d s=0 .
$$

The result now follows as in the proof of (i).

\section{AN AUXILIARY FUNCTION}

In this section, we shall prove a result analogous to Theorem 1 but where $\pi(T, \alpha)$ is replaced by the auxiliary function

$$
\psi(T, \alpha)=\sum_{\substack{l\left(\gamma^{\prime}\right) \leq T \\\left[\gamma^{\prime}\right]=\alpha}}^{\prime} \Lambda\left(\gamma^{\prime}\right),
$$

which can be related to the sums $\mathcal{S}_{T}(t)$ considered in the previous section. We shall adapt an approach used by Rousseau-Egele 18 to examine the quantity $\sigma^{k} T^{k / 2} e^{-h T} \psi(T, \alpha)$. For $a>0$, write $I(a)=[-a, a]^{k}$. Using the orthogonality relationship

we have that

$$
\frac{1}{(2 \pi)^{k}} \int_{I(\pi)} e^{-i\langle t, \alpha\rangle} e^{i\langle t, y\rangle} d t=\left\{\begin{array}{l}
1 \text { if } y=\alpha, \\
0 \text { if } y \in \mathbb{Z}^{k} \backslash \alpha,
\end{array}\right.
$$

$$
\psi(T, \alpha)=\frac{1}{(2 \pi)^{k}} \int_{I(\pi)} e^{-i\langle t, \alpha\rangle} \mathcal{S}_{T}(t) d t .
$$

Making the substitution $t \mapsto t / \sigma \sqrt{T}$, we obtain

$$
\sigma^{k} T^{k / 2} \psi(T, \alpha)=\frac{1}{(2 \pi)^{k}} \int_{I(\pi \sigma \sqrt{T})} e^{-i\langle t, \alpha\rangle / \sigma \sqrt{T}} \mathcal{S}_{T}(t / \sigma \sqrt{T}) d t .
$$

The next result is the key to the proof of Theorem 1.

\section{Proposition 5.}

$$
\lim _{T \rightarrow \infty} \sup _{\alpha \in \mathbb{Z}^{k}}\left|\frac{h \sigma^{k} T^{k / 2}}{e^{h T}} \psi(T, \alpha)-\frac{1}{(2 \pi)^{k / 2}} e^{-\left\langle\alpha, \mathcal{D}^{-1} \alpha\right\rangle / 2 T}\right|=0 .
$$

Using the identity

$$
e^{-\left\langle\alpha, \mathcal{D}^{-1} \alpha\right\rangle / 2 T}=\frac{1}{(2 \pi)^{k / 2}} \int_{\mathbb{R}^{k}} e^{i\langle t, \alpha\rangle / \sigma \sqrt{T}} e^{-\langle t, \mathcal{D} t\rangle / 2 \sigma^{2}} d t,
$$

we have established the bound

$$
\begin{aligned}
& (2 \pi)^{k}\left|\frac{h \sigma^{k} T^{k / 2}}{e^{h T}} \psi(T, \alpha)-\frac{e^{-\left\langle\alpha, \mathcal{D}^{-1} \alpha\right\rangle / 2 T}}{(2 \pi)^{k / 2}}\right| \\
& \leq\left|\int_{U(\delta \sigma \sqrt{T})} e^{-i\langle t, \alpha\rangle / \sigma \sqrt{T}}\left\{h e^{-h T} \mathcal{S}_{T}(t / \sigma \sqrt{T})-e^{-\langle t, \mathcal{D} t\rangle / 2 \sigma^{2}}\right\} d t\right| \\
& +\left|\int_{I(\pi \sigma \sqrt{T}) \backslash U(\delta \sigma \sqrt{T})} e^{-i\langle t, \alpha\rangle / \sigma \sqrt{T}} h e^{-h T} \mathcal{S}_{T}(t / \sigma \sqrt{T}) d t\right| \\
& +\left|\int_{\mathbb{R}^{k} \backslash U(\delta \sigma \sqrt{T})} e^{-i\langle t, \alpha\rangle / \sigma \sqrt{T}} e^{-\langle t, \mathcal{D} t\rangle / 2 \sigma^{2}} d t\right| \\
& =A_{1}(T, \alpha)+A_{2}(T, \alpha)+A_{3}(T, \alpha) .
\end{aligned}
$$


An easy calculation shows that

$$
\lim _{T \rightarrow \infty} \sup _{\alpha \in \mathbb{Z}^{k}} A_{3}(T, \alpha)=0,
$$

so, to complete the proof of Proposition 5, it remains to estimate $A_{1}(T, \alpha)$ and $A_{2}(T, \alpha)$. To do this we shall use the information on $s(t)$ and $\mathcal{S}_{T}(t)$ contained in Proposition 3 and Lemma 3.

Lemma 4. There exists $C>0$ such that, for all sufficiently small $\delta>0$,

$$
\lim _{T \rightarrow \infty} \sup _{\alpha \in \mathbb{Z}^{k}} A_{1}(T, \alpha) \leq C\left\{\int_{\mathbb{R}^{k}} e^{-\langle t, \mathcal{D} t\rangle / 4 \sigma^{2}} d t\right\} \delta^{2} .
$$

Proof. By Lemma 3, we have that, for $t \in U(\delta \sigma \sqrt{T})$,

$$
h e^{-h T} \mathcal{S}_{T}(t / \sigma \sqrt{T})=\frac{h e^{(s(t / \sigma \sqrt{T})-h) T}}{s(t / \sigma \sqrt{T})}+O\left(T^{-(k / 2+1)}\right) .
$$

Using the analyticity of $s(t)$ and the fact that $\nabla s(0)=0$, we have

$$
\left|e^{(s(t / \sigma \sqrt{T})-h) T}\left(\frac{h}{s(t / \sigma \sqrt{T})}-1\right)\right| \leq C \delta^{2} e^{-\langle t, \mathcal{D} t\rangle / 4 \sigma^{2}}
$$

for some constant $C>0$. Thus,

$$
\begin{aligned}
A_{1}(T, \alpha) & \leq \int_{U(\delta \sigma \sqrt{T})}\left|e^{(s(t / \sigma \sqrt{T})-h) T}-e^{-\langle t, \mathcal{D} t\rangle / 2 \sigma^{2}}\right| d t \\
& +C \delta^{2} \int_{U(\delta \sigma \sqrt{T})} e^{-\langle t, \mathcal{D} t\rangle / 4 \sigma^{2}} d t+O\left(\frac{1}{T}\right) .
\end{aligned}
$$

By Proposition 3, we know that $e^{(s(t / \sigma \sqrt{T})-h) T}$ converges to $e^{-\langle t, \mathcal{D} t\rangle / 2 \sigma^{2}}$, as $T \rightarrow \infty$. Furthermore, we have the estimate

$$
\left|e^{(s(t / \sigma \sqrt{T})-h) T}-e^{-\langle t, \mathcal{D} t\rangle / 2 \sigma^{2}}\right| \leq 2 e^{-\langle t, \mathcal{D} t\rangle / 4 \sigma^{2}} .
$$

Hence, applying the Dominated Convergence Theorem, we obtain the desired result.

\section{Lemma 5.}

$$
\lim _{T \rightarrow \infty} \sup _{\alpha \in \mathbb{Z}^{k}} A_{2}(T, \alpha)=0 .
$$

Proof. By Lemma 3(ii), for $t \notin U(\delta / \sigma \sqrt{T})$,

$$
e^{-h T} \mathcal{S}_{T}(t / \sigma \sqrt{T})=O\left(T^{-(k / 2+1)}\right),
$$

so that $\sup _{\alpha \in \mathbb{Z}^{k}} A_{2}(T, \alpha)=O\left(T^{-1}\right)$.

Proof of Proposition 5. Combining the above results we have that

$\lim _{T \rightarrow \infty} \sup _{\alpha \in \mathbb{Z}^{k}}\left|\frac{h \sigma^{k} T^{k / 2}}{e^{h T}} \psi(T, \alpha)-\frac{1}{(2 \pi)^{k / 2}} e^{-\left\langle\alpha, \mathcal{D}^{-1} \alpha\right\rangle / 2 T}\right| \leq C\left\{\int_{\mathbb{R}^{k}} e^{-\langle t, \mathcal{D} t\rangle / 4 \sigma^{2}} d t\right\} \delta^{2}$.

Since this holds for all sufficiently small $\delta>0$, the proof is complete. 


\section{Proof of Theorem 1}

In this section we will use elementary arguments to deduce Theorem 1 from Proposition 5. Whenever we make a big- $O$ estimate, the implied constant will be independent of $\alpha$.

Write

$$
\psi^{*}(T, \alpha)=\sum_{\substack{l(\gamma) \leq T \\[\gamma]=\alpha}} l(\gamma) .
$$

An easy argument shows that

$$
\psi(T, \alpha)=\psi^{*}(T, \alpha)+O\left(T^{2} e^{h T / 2}\right) .
$$

Thus, Proposition 5 implies the following.

\section{Proposition 6.}

$$
\lim _{T \rightarrow \infty} \sup _{\alpha \in \mathbb{Z}^{k}}\left|\frac{h \sigma^{k} T^{k / 2}}{e^{h T}} \psi^{*}(T, \alpha)-\frac{1}{(2 \pi)^{k / 2}} e^{-\left\langle\alpha, \mathcal{D}^{-1} \alpha\right\rangle / 2 T}\right|=0 .
$$

Finally, we consider $\pi(T, \alpha)$. It is easy to see that

$$
\psi^{*}(T, \alpha) \leq T \pi(T, \alpha) .
$$

For the corresponding lower bound, choose $\tau>0$ and set $\theta=(1+\tau)^{-1}<1$. Then

$$
\begin{aligned}
\frac{T \pi(T, \alpha)}{e^{h T}} & =\frac{T}{e^{h T}} \sum_{\substack{\theta T<l(\gamma) \leq T \\
[\gamma]=\alpha}} 1+\frac{T \pi(\theta T, \alpha)}{e^{h T}} \\
& \leq \frac{1+\tau}{e^{h T}} \sum_{\substack{\theta T<l(\gamma) \leq T \\
[\gamma]=\alpha}} l\left(\gamma^{\prime}\right)+\frac{T \pi(\theta T, \alpha)}{e^{h T}} \\
& \leq \frac{(1+\tau) \psi^{*}(T, \alpha)}{e^{h T}}+\frac{T \#\{\gamma: l(\gamma) \leq \theta T\}}{e^{h T}} .
\end{aligned}
$$

Using the estimate $\#\{\gamma: l(\gamma) \leq T\}=O\left(e^{h T} / T\right)$ [13], we have established

$$
\begin{aligned}
0 & \leq \frac{T^{k / 2+1}}{e^{h T}} \pi(T, \alpha)-\frac{T^{k / 2}}{e^{h T}} \psi^{*}(T, \alpha) \\
& \leq \frac{\tau T^{k / 2}}{e^{h T}} \psi^{*}(T, \alpha)+O\left(T^{k / 2} e^{(\theta-1) h T}\right),
\end{aligned}
$$

so that, by applying Proposition 6 ,

$$
\limsup _{T \rightarrow \infty} \sup _{\alpha \in \mathbb{Z}^{k}}\left|\frac{T^{k / 2+1}}{e^{h T}} \pi(T, \alpha)-\frac{T^{k / 2}}{e^{h T}} \psi^{*}(T, \alpha)\right| \leq \frac{\tau}{(2 \pi)^{k / 2} h \sigma^{k}} .
$$

Since $\tau>0$ is arbitrary, this proves Theorem 1 .

\section{Homologically full Anosov flows}

The asymptotic identity (0.1) has been generalized to certain transitive Anosov flows $\phi_{t}: N \rightarrow N$, where $N$ is a compact smooth Riemannian manifold. We now use $\gamma$ to denote a (prime) periodic orbit of $\phi$, with least period $l(\gamma)$. Once again, we write $[\gamma]$ for the torsion-free part of the homology class of $\gamma$ in $H_{1}(N, \mathbb{Z}) \cong \mathbb{Z}^{k} \oplus$ Tor. We say that $\phi$ is homologically full if every homology class in $H_{1}(N, \mathbb{Z})$ is represented 
by a closed orbit. In this case, there exist $\xi \in H^{1}(N, \mathbb{R}), 0<h^{*} \leq h$ and $C_{0}>0$ such that

$$
\pi(T, \alpha) \sim C_{0} e^{-\langle\xi, \alpha\rangle} \frac{e^{h^{*} T}}{T^{k / 2+1}}, \quad \text { as } T \rightarrow \infty .
$$

This result was first proved in [19], drawing on ideas from [10]. An alternative proof was given in 2 and a more precise version is contained in [17. In this section, we shall sketch a new proof of (5.1), using the techniques discussed above. However, we will not make any claims about uniformity.

Remark. We can define a function $\mathfrak{p}: H^{1}(N, \mathbb{R}) \rightarrow R$ by $\mathfrak{p}([\omega])=P(\omega(\mathcal{X}))$, where $\omega$ is a closed 1-form representing the cohomology class $[\omega]$ and $\mathcal{X}$ is the vector field tangent to $\phi$. Then $\xi$ and $h^{*}$ are characterized by the formulae

$$
h^{*}=\mathfrak{p}(\xi)=\min \left\{\mathfrak{p}\left(\xi^{\prime}\right): \xi^{\prime} \in H^{1}(N, \mathbb{R})\right\} .
$$

We begin by considering a modified family of $L$-functions. We define

$$
L(s, t)=\prod_{\gamma}\left(1-e^{-s l(\gamma)+\langle\xi, \alpha\rangle+i\langle t,[\gamma]\rangle}\right)^{-1},
$$

which converges for $\operatorname{Re}(s)>h^{*}$. The extension of $L^{\prime} / L$ to a uniform strip, described in Propositions 1 and 2, is no longer valid; however, the next result provides a weaker substitute. As in the case of closed geodesics, an analysis due to Dolgopyat [5] is crucial here. For $\rho>0$, write

$$
\mathcal{R}(\rho)=\left\{s: \operatorname{Re}(s)>h^{*}-|\operatorname{Im}(s)|^{-\rho},|\operatorname{Im}(s)| \geq 1\right\} .
$$

Proposition 7 ([17). There exists a constant $\rho>0$ such that, for all sufficiently small $\delta>0$, the following statements are true.

(i) There exists an analytic function $s: U(\delta) \rightarrow\left\{z \in \mathbb{C}: \operatorname{Re}(z) \leq h^{*}\right\}$, satisfying $s(0)=h^{*}$ and $\operatorname{Re}(s(t))<h^{*}$ for $t \neq 0$ such that

$$
\frac{L^{\prime}(s, t)}{L(s, t)}+\frac{1}{s-s(t)}
$$

is analytic in $\mathcal{R}(\rho)$.

(ii) For $t \notin U(\delta), L^{\prime}(s, t) / L(s, t)$ is analytic in $\mathcal{R}(\rho)$.

Proposition 8 ([17]). There exist $C>0$ and $\beta>0$ such that, for all $t \in[-\pi, \pi]^{k}$,

$$
\left|\frac{L^{\prime}(s, t)}{L(s, t)}\right| \leq C|\operatorname{Im}(s)|^{\beta},
$$

for $s \in \mathcal{R}(\rho)$.

Although the function $s(t)$ is now complex valued, it is still the case that $\nabla s(0)=$ 0 and that $\nabla^{2} s(0)$ is real and strictly negative definite. Moreover, again writing $\mathcal{D}=-\nabla^{2} s(0)$ and $\sigma^{2 k}=\operatorname{det} \mathcal{D}$, the function $e^{s(t)-h^{*}}$ still satisfies the conclusions of Proposition 3.

We shall now mimic the arguments of Section 2. However, the weaker bounds on $L^{\prime}(s, t) / L(s, t)$ force us to use a more complicated auxiliary function. For $n \geq 0$, define

$$
\psi_{n}(T, \alpha)=\frac{e^{\langle\xi, \alpha\rangle}}{n !} \sum_{\substack{\left.l\left(\gamma^{\prime}\right) \leq T \\ \gamma^{\prime}\right]=\alpha}}^{\prime} \Lambda\left(\gamma^{\prime}\right)\left(e^{h^{*} T}-e^{h^{*} l\left(\gamma^{\prime}\right)}\right)^{n} .
$$


Then we have the identity

$$
\sigma^{k} T^{k / 2} \psi_{n}(T, \alpha)=\frac{1}{(2 \pi)^{k}} \int_{I(\pi \sigma \sqrt{T})} e^{-i\langle t, \alpha\rangle / \sigma \sqrt{T}} \mathcal{S}_{T}^{*}(t / \sigma \sqrt{T}) d t,
$$

where

$$
\mathcal{S}_{T}^{*}(t)=\sum_{l\left(\gamma^{\prime}\right) \leq T}^{\prime} \Lambda\left(\gamma^{\prime}\right) e^{\left\langle\xi,\left[\gamma^{\prime}\right]\right\rangle+i\left\langle t,\left[\gamma^{\prime}\right]\right\rangle}\left(e^{h^{*} T}-e^{h^{*} l\left(\gamma^{\prime}\right)}\right)^{n} .
$$

In order to estimate the function $\mathcal{S}_{T}^{*}(t)$ we need the following identity, for $d>h^{*}$,

$$
\mathcal{S}_{T}^{*}(t)=\frac{1}{2 \pi i} \int_{d-i \infty}^{d+i \infty}\left(-\frac{L^{\prime}(s, t)}{L(s, t)}\right) \frac{e^{(s+n) T}}{s(s+1) \cdots(s+n)} d s,
$$

where we have used the formula

$$
\frac{1}{2 \pi i} \int_{d-i \infty}^{d+i \infty} \frac{y^{s}}{s(s+1) \cdots(s+n)} d s=\left\{\begin{array}{l}
0 \text { if } 0<y<1 \\
\frac{1}{n !}\left(1-\frac{1}{y}\right)^{n} \text { if } y \geq 1 .
\end{array}\right.
$$

Choose $0<\epsilon<1 / \rho$ and set $R=T^{\epsilon}$ and $d=h^{*}+T^{-1}$. Then replacing the integral in (5.3) with the truncated integral $\int_{d-i R}^{d+i R}$ introduces an error of order $O\left(e^{\left(h^{*}+n\right) T} / T^{\epsilon n}\right)$. Using the estimates

(a)

$$
\begin{aligned}
& \left|\left(\int_{h^{*}-R^{-\rho}+i R}^{d+i R}+\int_{h^{*}-R^{-\rho}-i R}^{d-i R}\right)\left(-\frac{L^{\prime}(s, t)}{L(s, t)}\right) \frac{e^{(s+n) T}}{s(s+1) \cdots(s+n)} d s\right| \\
& =O\left(R^{\beta-\rho-n-1} e^{\left(h^{*}+n\right) T}\right)=O\left(e^{\left(h^{*}+n\right) T} T^{-\epsilon(\rho+n+1-\beta)}\right) ;
\end{aligned}
$$

(b)

$$
\begin{aligned}
& \left|\int_{h^{*}-R^{-\rho} \pm i}^{h^{*}-R^{-\rho} \pm i R}\left(-\frac{L^{\prime}(s, t)}{L(s, t)}\right) \frac{e^{(s+n) T}}{s(s+1) \cdots(s+n)} d s\right| \\
& =O\left(R^{\beta} e^{\left(h^{*}+n-R^{-\rho}\right) T}\right)=O\left(T^{\beta \epsilon} e^{\left(h^{*}+n\right) T} e^{-T^{1-\epsilon \rho}}\right),
\end{aligned}
$$

we may repeat the proof of Lemma 3 to obtain the following lemma.

Lemma 6. Setting $N=\min \{\epsilon n, \epsilon(\rho+n+1-\beta)\}$, we have the following. (The implied constants are independent of $t$.)

(i) For $t \in U(\delta)$,

$$
\mathcal{S}_{T}^{*}(t)=\frac{e^{(s(t)+n) T}}{s(t)(s(t)+1) \cdots(s(t)+n)}+O\left(\frac{e^{\left(h^{*}+n\right) T}}{T^{N}}\right) .
$$

(ii) For $t \notin U(\delta), \mathcal{S}_{T}^{*}(t)=O\left(e^{\left(h^{*}+n\right) T} / T^{N}\right)$.

Provided $n$ is sufficiently large such that $N>k / 2$, we may repeat the arguments used in the proof of Proposition 5 to obtain

$$
\lim _{T \rightarrow \infty} \sup _{\alpha \in \mathbb{Z}^{k}}\left|\prod_{j=0}^{n}\left(h^{*}+j\right) \frac{\sigma^{k} T^{k / 2}}{e^{\left(h^{*}+n\right) T}} \psi_{n}(T, \alpha)-\frac{1}{(2 \pi)^{k / 2}} e^{-\left\langle\alpha, \mathcal{D}^{-1} \alpha\right\rangle / 2 T}\right|=0 .
$$

From this it immediately follows that

$$
\psi_{n}(T, \alpha) \sim \frac{1}{(2 \pi)^{k / 2} \sigma^{k}} \prod_{j=0}^{n} \frac{1}{\left(h^{*}+j\right)} \frac{e^{\left(h^{*}+n\right) T}}{T^{k / 2}}, \quad \text { as } T \rightarrow \infty .
$$


The asymptotic formula

$$
\psi_{0}(T, \alpha) \sim \frac{1}{(2 \pi)^{k / 2} h^{*} \sigma^{k}} \frac{e^{h^{*} T}}{T^{k / 2}}, \quad \text { as } T \rightarrow \infty,
$$

now follows by a standard inductive argument (cf. p. 35 of [7]). Finally, (5.1) may be deduced as in Section 3. (Note that one needs the a priori estimate $\lim \sup _{T \rightarrow \infty}(\pi(T, \alpha))^{1 / T} \leq e^{h^{*}}$, which follows from the convergence of (5.2) for $\operatorname{Re}(s)>h^{*}$.)

\section{REFERENCES}

1. N. Anantharaman, Precise counting results for closed orbits of Anosov flows, Ann. Sci. École Norm. Sup. 33 (2000), 33-56. MR 2002c:37048

2. M. Babillot and F. Ledrappier, Lalley's theorem on periodic orbits of hyperbolic flows, Ergodic Theory Dyn. Syst. 18 (1998), 17-39. MR 99a:58128

3. M. Babillot and M. Peigné, Homologie des géodésiques fermées sur des variétés hyperboliques avec bouts cuspidaux, Ann. Sci. École Norm. Sup. 33 (2000), 81-120. MR 2001b:37043

4. D. Dolgopyat, On decay of correlations for Anosov flows, Annals of Math. 147 (1998), 357390. MR 99g:58073

5. D. Dolgopyat, Prevalence of rapid mixing in hyperbolic flows, Ergodic Theory Dyn. Syst. 18 (1998), 1097-1114. MR 2000a:37014

6. C. Epstein, Asymptotics for closed geodesics in a homology class, the finite volume case, Duke Math. J. 55 (1987), 717-757. MR 89c:58098

7. A. Ingham, The Distribution of Prime Numbers, Cambridge University Press, Cambridge, 1990; Reprint of the 1932 original. MR 91f:11064

8. A. Katsuda, Density theorems for closed orbits, Geometry and analysis on manifolds (T. Sunada, ed.), Lecture Notes in Mathematics 1339, Springer, Berlin, 1988, pp. 182-202. MR 89j:58066

9. A. Katsuda and T. Sunada, Homology and closed geodesics in a compact Riemann surface, Amer. J. Math. 110 (1988), 145-155. MR 89e:58093

10. A. Katsuda and T. Sunada, Closed orbits in homology classes, Inst. Hautes Études Sci. Publ. Math. 71 (1990), 5-32. MR 92m:58102

11. M. Kotani, A note on asymptotic expansions for closed geodesics in homology classes, Math. Ann. 320 (2001), 507-529. MR 2002h:58044

12. S. Lalley, Closed geodesics in homology classes on surfaces of variable negative curvature, Duke Math. J. 58 (1989), 795-821. MR 91a:58143

13. W. Parry and M. Pollicott, Zeta functions and the periodic orbit structure of hyperbolic dynamics, Astérisque 187-188 (1990), 1-268. MR 92f:58141

14. R. Phillips and P. Sarnak, Geodesics in homology classes, Duke Math. J. 55 (1987), 287-297. MR 88g:58151

15. M. Pollicott, Homology and closed geodesics in a compact negatively curved surface, Amer. J. Math. 113 (1991), 379-385. MR 92e:58158

16. M. Pollicott and R. Sharp, Exponential error terms for growth functions on negatively curved surfaces, Amer. J. Math. 120 (1998), 1019-1042. MR 99h:58148

17. M. Pollicott and R. Sharp, Asymptotic expansions for closed orbits in homology classes, Geom. Dedicata 87 (2001), 123-160. MR 2003b:37051

18. J. Rousseau-Egele, Un théorème de la limite locale pour une classe de transformations dilatantes et monotones par morceaux, Ann. Probab. 11 (1983), 772-788. MR 84m:60032

19. R. Sharp, Closed orbits in homology classes for Anosov flows, Ergodic Theory Dyn. Syst. 13 (1993), 387-408. MR 94g:58169

20. G. Tenenbaum, Introduction to Analytic and Probabilistic Number Theory, Cambridge University Press, Cambridge, 1995. MR 97e:11005b

Department of Mathematics, University of Manchester, Oxford Road, Manchester M13 9PL, United Kingdom

E-mail address: sharp@maths.man.ac.uk 